\title{
Design and Realization of the computer system based on 3D Virtual Tourism
}

\author{
HUANG Liusuo ${ }^{1,}$, , SONG Yan² \\ ${ }^{1,2}$ Department of Information Technology, Henan Institute of Eduaction, Henan ZhengZhou \\ 450046,china \\ aemail: huangliusuo@163.com
}

Keywords: Virtual reality; circular fisheye image; image mosaic; panorama roaming

\begin{abstract}
VR is a integrated technology, involving computer graphics, human-computer interaction technology, sensor technology, artificial intelligence and other areas, it use computer generation vivid 3D view, listen to, smell and feeling, make the person as a participant through proper device, naturally in the virtual world into the experience and interact. When the user moves the position, the computer can immediately carry out complex operations, the exact 3D world image sent back to produce a sense of presence. The technique integrates computer graphics (CG) technology, computer simulation technology, artificial intelligence, sensor technology, display technology, network parallel processing technology of the latest achievements, a simulation system of high technology aided by computer technology generation. In Wuhan University of science and technology, South environment as a virtual space, shooting teaching building, library, gymnasium, living quarters, roads, trees, gardens, pavilions and other outdoor scenes generated panorama, virtual campus roaming, and for the user to provide keyboard, mouse and other control means and way of human-computer interaction.
\end{abstract}

\section{Introduction}

Schools in order to show their own style, more and more attention to publicity, the need for a more direct way to promote their own. Virtual reality technology is applied to the school, is the virtual campus, can display all kinds of the school's software and hardware environment. In this paper, we discuss the technology of panoramic roaming, and think that the three problems need to be solved in the panorama roaming technology: the creation of the panorama, the editing of the roaming space and the design of the browser. In the panoramic roaming technology study based on the two round fish eye image of the spherical panorama generation method is generated for each of the scenes of the spherical panorama based on, in order to achieve a moderately complex degree, has certain ability of human-computer interaction panoramic campus roaming system.

Advanced in the system, the weakest link is the campus electronic information construction, although in the overall or partial realization automation, but on the whole campus information no a three-dimensional, image display, only through the basic text page, add on a few pieces of photos, a display of school, such not only to school oneself image construction drag, on the other hand, is not conducive to the students and parents on the campus environment is a rapid and comprehensive understanding to spend a lot of time and energy to foot measurement.

The Ministry of education in a series of related documents, repeatedly comes to the virtual campus, expounds the status and function of virtual campus. The specific application of virtual campus virtual reality technology is the earliest in the education and training, it is from shallow to deep three application level, respectively, to adapt to the school demand in different degree: simple virtual environment of our school for visitors to browse the campus teaching and educational life, based on the virtual campus 3D visualization function relatively complete with students as the center, adding a series of user-friendly features, with virtual reality technology as distance education platform based on virtual reality, virtual distance education, college enrollment can be set to the campus and distance education teaching for mobile electronic teaching place, through interactive distance learning course catalog and website, the tools for the campus LAN link to the 
site, continuing education can provide long-distance open, to each terminal, but also for the community to provide new and high technology Such as occupation training opportunities, create greater economic benefits and social benefits[1].

With the continuous development and improvement of virtual reality technology, and hardware equipment prices continue to lower, we believe that virtual reality technology with its powerful teaching advantages and potential will gradually be educators attention and favor, eventually in the education and training of a wide range of fields and play an important role. This paper is the study of how to use the existing computer technology, campus plan or pictures to produce a stereoscopic form showing films, and even can realize the virtual campus roaming, undertake the functions of panoramic campus. Based on the principle of fisheye image, abstract to graphic photographs, in the seamless splicing picture realize fixed-point rotation display, is the flash ActionScript language a more in-depth understanding, using pv3d base class, powerful virtual campus roaming results.

\section{Virtual Reality Technology}

Virtual reality is the people through the computer for complex data to a new way of visualization and interactive, compared with the traditional man-machine interface, as well as the popular windows operating, virtual reality on the thought of technology have a qualitative leap. Virtual reality of "reality" is refers to the physical or functional significance exists in the world any things or the environment, it can be practically achievable, can also is actually difficult to achieve or impossible.

And "virtual" is the meaning of the computer generated. Therefore, the virtual reality refers to a special computer generated environment, human beings may be through the use of all kinds of special devices will be their "projection" to the environment, and operation and control environment, achieve the purpose of the special, that man is the master of this environment.

In essence, virtual reality is a kind of advanced computer user interface through to the user while providing such as visual, auditory, tactile and other various intuitive and natural perception of real-time interactive means, to maximize the then user operation. Depending on the application of virtual reality technology, its role can be performance for different forms, such as a concept design or idea visualization and operability, realistic remote field effect, achieve arbitrarily complex environment of cheap simulation for training purposes etc..

The so-called multi perception is that in addition to the general computer technology has the visual perception, there are auditory perception, force perception, tactile perception, motion perception, and even taste perception, smell perception, etc.. The ideal virtual reality technology should have the function of perception. Because of the limitation of the technology, especially the sensing technology, the virtual reality technology has the function of perception, such as vision, hearing, force sense, touch, movement and so on.

Immersion, also known as the presence of a sense, refers to the user as the protagonist exists in the simulation environment of the real degree. Ideal simulation environment should users is difficult to distinguish between true and false, enables the user to dedicate to the computer to create a 3D virtual environment, everything in the environment appears to be true, sounds really, moving up is really, even smell and taste, everything feels really, like in the real world[2].

The natural degree of the user's ability to operate and get feedback from the environment (including real time). For example, a user can hand to direct simulation grab virtual objects in the environment, then hand to hold things feeling, and can be object feel the weight of, was caught in the view of the object can also immediately with the movement of the hand and mobile.

Stressed that the virtual reality technology has broad space to imagine, broaden the scope of human cognition, not only can represent the real environment, also can freely idea objective does not exist or even impossible environment. Generally speaking, a complete virtual reality system by virtual environment, high performance computer virtual environment processor core, with head mounted display (HMD) as the core of visual system, as the core of auditory system, by azimuth tracking device, data glove and inserted into the body of the azimuth tracking devices, and taste, smell, touch and force sleep feedback system functional modules with voice recognition and voice 
synthesis and sound localization.

Here, the virtual environment processor is the heart of the VR system, to complete the production and processing functions of the virtual world. Input devices provide input to the VR system from the user, and allow users to change their location, line of sight and visual field in the virtual environment, but also allows to change the location and direction of virtual objects in the virtual environment. While the output device is the VR system to the virtual environment of the integrated production of the various sensory information output to the user, the user generates an immersive sense of reality.

Virtual environment is the core content of virtual reality technology. The goal of dynamic environment modeling technology is to obtain the 3D data of the actual environment, and to establish the corresponding virtual environment model according to the needs of the application. 3D data can be obtained by using CAD technique (rules), and more environmental needs based on visual modeling technique of non-contact, the organic combination of the two can effectively improve the efficiency of data acquisition.

The key to the application of virtual reality is to find the appropriate occasions and objects, that is, how to play the imagination and creativity. Choosing appropriate application objects can greatly improve the production efficiency, reduce labor intensity and improve the quality of product development. In order to achieve this goal, it is necessary to study the development of virtual reality tools. For example, virtual reality system development platform, distributed virtual reality technology, etc[3].

\section{Rich application interface function}

The 3D virtual campus system has good openness, provides support for other information, the system can be directly embedded into the university website, can be connected to the touch screen, can also be connected to the video monitoring system.

Campus network in each department or other departments can through the virtual campus of embedded code, to produce various forms of simulation bulletin board, such as campus message board, enrollment information, etc., multistate advertising from the network simulation, with extremely interesting and novel, greatly increase click through rate, especially for the celebration activities, and more excellent propaganda effect. For example, the website published notice of the meeting or the exam location information can be embedded location code, users in the browse information content and display in the virtual campus to see the specific location of the meeting or the examination, improve the University's reputation, contribute to the height of the University's own propaganda and information concentration, configuration and interaction.

The first step: boot flash 8.0, select the menu "modify, document" command to open the document properties box, the width of the document set for 450px, height setting to be 250px and background color to black; selection menu "File > import, import to the library..." Command in the dialog that appears, select the static panorama, and then click "open" button to import the picture, select menu "Insert > time axis, layer" command to insert three new layer, double click on the layer name can be renamed, from top to bottom were named:, control script, a control button and a display area, a panorama[4].

The second step: select the menu to insert new elements..." Dialog box appears, select the type of "button", the name is "zoom", this creates a zoom button, then using this method, and then make a zoom out button; create a named "response" button, the button is a rectangle, is about the size of the $360 * 200$; select the menu "Insert > new component..." Dialog box appears, select the type of "clip", the name "panorama"; choose the menu window > Library "open" library ", the picture (panorama. JPG) and drop it onto the stage and copy a, their splicing.

The third step: open the "library", the three button onto the control button "layer first frame on the stage of the" response "display area in panorama button position (probably in the center of the scene), and click the" properties "button below the stage, the transparency of the set (Alpha)" 0 ", the width of 360 , height is $200, \mathrm{X}$ is $50, \mathrm{Y}$ is $25 ; "$ zoom "and" zoom "button in the lower right button response", again in the twenty-fifth frame of the layer and press the F5 key is inserted into 
the frame; in the "display area" layer first frame on the stage of a drawing with a response of "button" and other large rectangular, just covered in "response" button, the "library" in the "panorama stitching" film drop to first frames "panorama", in the last twenty-fifth frames of the two layer of press F5.

Step 4: "panorama" film is selected and click below the "properties" button, in fact, the example set to "to"; in the control script "layer 1 frame press F6 insert the key frames, click below the stage of the actions panel and add following scripts:

$\mathrm{L}=459 ; / / \mathrm{L}$ is the width of the scene

$\mathrm{H}=250$; //h is the height of the scene

$\mathrm{T}=50 ; / / \mathrm{t}$ is displayed at the top of the coordinates

$\mathrm{B}=250 ; / / \mathrm{b}$ is displayed at the bottom of the area coordinate

$\mathrm{V}=250 ; / / \mathrm{v}$ is the horizontal line coordinates

$\mathrm{M}=(\mathrm{t}+\mathrm{b}) / 2 ; / / \mathrm{m}$ is the center coordinate of the region

$\mathrm{Si}=100 *(\mathrm{~B}-\mathrm{T}) /$ (tupian._height); //si is the minimum zoom

Note: "/ /" behind the statement that is part of the data here, according to the actual size of the production set.

The fifth step: in the 5 frames "scripting" layer in the press the F6 key, and then open the panel of the "action", input: "stop ();" (Note: quotes don't input) script; in the 10th frame press F6, and then open the "action" input panel script (for details refer to the source code), the script is mainly to obtain the cursor position. According to the moving direction and speed of the cursor, control the direction and velocity of the panorama. Press the F6 button in the eleventh frame, open the "action" panel, enter: "gotoAndPlay (10);", it is mainly to achieve repeated implementation of the two frame code, so as to ensure the mobile mouse, the panorama has been maintained.

Step 6: in the 15 frames "scripting" layer in the press the F6 key, and then open the "action" input panel script (for details refer to the source code), the script is mainly in proportion to change panorama of height and width, so as to realize the amplification effect. Press the F6 button in the sixteenth frame, open the "action panel", enter: "gotoAndPlay (15);", the role is to repeat the implementation of the fifteenth frame script.

Step 7: in the "scripting" layer of the 20th frame press F6, then add a script (for details refer to the source code), the script is let the picture after the amplification can be reduced, and set reduction cannot be less than the original size. Finally in the twenty-first frame press F6 key, plus the script: "gotoAndPlay (20);", the role is to repeat the implementation of the twentieth frame script.

Step 8: click layer "display area", then right-click and choose "Mask" (as shown in Figure 3-9), so the panorama can only appear in the display area; "zoom" button is selected, open the action "input panel script (for details refer to the source code), the script is when press the left button of the mouse, the 15 frame code execution, namely amplify panorama, release the left mouse button to execute the 5th frame code, stop code execution[5].

\section{Conclusion}

In the design and development of the whole functional system, I have encountered many problems. Shots photos from the beginning and need to consider many aspects, the choice of attractions and surface range, and framing in people, objects and scenery if you need to join, and is related to public privacy issues in the treatment of a series of problems.

Then it is from the shot back hundreds of pictures to choose the need to splice, as far as possible to do not leave a trace after stitching, this is a very careful and very mechanical work. After processing a good picture, making a panorama, the next is how to use the FLASH script to achieve a 360 degree panoramic display.

This topic for me, everything is new, in order to complete the graduation design, I in the online search a lot of things, and friends exchange, learn from each other, something about flash3d, any for me are strange.

There is flash3d component installation and first from the Google open source project download file corresponding to the MXP package using Adobe extension manager to install the extension, to 
note here is version of the problem, corresponding to the flash CS3 is version 1.8, corresponding to the Flash CS4 is version 2.0, I made this mistake, version is not clear. There is the need to re position the flash run after the need to introduce the absolute path of the class package, otherwise the compiler will be wrong, the lack of the corresponding XX class.

\section{Acknowledgement}

Digital Textbook Research. Henan Provincial Social Science Planning and decision-making advisory commissioned project.2015JCWT06.

\section{References}

[1] Li Guilan et al. The new concept of EQ and non intelligence training Tibet people's Publishing House 2001.9.

[2] Tan Zhenhua: "teaching theory of reading" social science literature press, 2000.

[3] Hu Chundong: "English teaching method", higher education press.

[4] Wang Cairen Jia Guanjie Hu Chundong, editor in chief: "foreign language educational psychology" Guangxi Education Publishing House.

[5] Liu Jianhua, editor in chief: "high school English teaching innovation" full, Academic Press. 